\title{
A Hesitant Fuzzy Multiple Attribute Decision Making Method Based on Complementary Judgment Matrix
}

\author{
Jibin Lan, Wenqian Kang and Mingming $\mathrm{Hu}^{*}$ \\ Business School, Guangxi University, Nanning, Guangxi 530004, P.R. China \\ ${ }^{*}$ Corresponding author
}

\begin{abstract}
Hesitant fuzzy set allow an element in the domain with a set of membership degree between 0 and 1 , which is a powerful tool to express the uncertainty alternative assessment value of decision makers. A hesitant fuzzy multiple attribute decision making method is proposed based on additive consistency theory of fuzzy complementary judgment matrix. First, we define mean residual between two hesitant fuzzy elements and a new fuzzy complementary judgment matrix and then prove it has additive consistency; on this basis, we gain a new weighted fuzzy complementary judgment matrix. Then, the fuzzy complementary judgment matrix is implemented to rank and select alternatives. Finally, an example is given to illustrate the feasibility and effectiveness of the proposed method.
\end{abstract}

Keywords—hesitant fuzzy set;fuzzy complementary judgment matrix; additive consistency;multiple attribute decision making

\section{INTRODUCTION}

Since the fuzzy sets proposed by Zadeh [1], it has been brought into focus on dealing with uncertainty problem. Then, some extension of fuzzy sets have been introduced because of the complexity and uncertainty of real life. Thereinto, type- 2 fuzzy set that allows the membership of a given element as a fuzzy set proposed by Zadeh [2]; Dubois and Prade [3] presented n-type fuzzy set [3] which generalized 2-type fuzzy set; fuzzy multiple set introduced by Yager [4], in which the elements can be repeated more than once; Atanassov [5] presented the concept of intuitionistic fuzzy set (IFS) and analyzed the fuzzy set by constructed membership function, non-membership function and hesitancy function.

In the process of the ideal decision making, rational individual thinking and tacit understanding group thinking are the key to form consistent accuracy judgment. However, human limited knowledge and limited logos make it hard for experts to give a precise, satisfy consistency conditions, the overall optimal judgment information, always hesitate in several possible values [6]. As an extension of fuzzy set, Torra [7] proposed the concept of hesitant fuzzy set in which the membership degree of an element in the domain has a set of possible values between 0 and 1 . Proposed concept is a powerful tool to deal imprecise and uncertain decision making problem. Currently, hesitant fuzzy multiple attribute decision making problem has caused wide attention of scholars both at home and abroad and has achieved phased research results. Many kinds of hesitant fuzzy aggregation operators [8, 9, 10], distance measures and similarity measures [11, 12, 13] have been studied to deal with multiple attribute decision making problem. Xia et al. [14] proposed a set of hesitant fuzzy information fusion operators and discussed their generalizations. $\mathrm{Xu}$ et al. $[15,16]$ introduced some basic concepts of hesitant fuzzy set such as correlation coefficient, entropy, cross entropy and so on and applied them to the multiple attribute decision making. Wei [17] presented a set of hesitant fuzzy prioritized operators in multiple attribute decision making. Xia et al. [18] and Zhang [19] researched the application of hesitant fuzzy aggregation operator in multiple attribute decision making. Farhadinia [20, 21] proposed the formula of distance, similarity degree, entropy on hesitant fuzzy set and a new method for ranking alternatives expressed with hesitant fuzzy set. The consistency of the preference relationship will have a direct effect on the rationality of the final ranking of the alternatives, and the lack of consistency will produce the result that is not logical. Recently, increasing scholars have come to research the multiple attribute decision making problem which has consistency. Zhang et al. [22] develop a consistency- improving process to adjust unacceptably multiplicative consistency into an acceptably multiplicative one. Zhu [23] develop some consistency measures for HFLPRs to ensure that the DMs are being neither random nor illogical. Zhang et al. [24] introduce the acceptable consistency of the PLPR and use aggregation operators to obtain the comprehensive preference values of alternatives and then rank the alternatives. Tan et al. [25] constructed an optimization model based on the maximization of group consistency index to research the multiple attribute decision making problem. Rank and select alternatives is the purpose of solving multiple attribute decision making problem. At present, The common methods including aggregation operators [26, 27, 28, 29], TOPSIS[30, 31, 32, 33] , VIKOR[34, 35] , grey relational analysis[36, 37] et al. methods. This paper research the additive consistency of hesitant fuzzy preference relation by constructed a hesitant fuzzy complementary judgment matrix and then proposed a new approach to analyze hesitant fuzzy multiple attribute decision making problem.

The remaining sections of this paper are set up as follows: section 2 reviews and fixes basic concepts; section 3 constructs a new fuzzy complementary judgment matrix and proves it has additive consistency and then it's generalization is generalized; section 4, a new approach to multiple attribute decision making under hesitant fuzzy environment is developed; section 5 provides a numerical example to demonstrate the feasibility and validity of the proposed method and in section 6 conclusions are gives. 


\section{PRELIMINARIES}

In this section, we will give some basic definitions of hesitant fuzzy set, fuzzy complementary judgment matrix, fuzzy complementary judgment matrix with additive consistency.

\section{A. Hesitant Fuzzy Set}

Limited knowledge and logos of people make it hard for experts to give a consistency condition. Based on this, Torra [7] proposed the concept of hesitant fuzzy set as follow:

Definition 1. [7] let $X$ be a reference set. An hesitant fuzzy set (HFS) $E$ on $X$ is defined in terms of a function $h_{E}(x)$ that returns a subset of $[0,1]$ when applied to $X$.

For convenience, the HFS can be expressed by Wei [17] mathematically as follow:

$$
E=\left\{<x, h_{E}(x)>\mid x \in X\right\}
$$

where $h_{E}(x)$ is a set of values in $[0,1]$, which represent the possible membership degrees of the element $x \in X$ to $E$. For convenience, we call $h_{E}(x)$ a hesitant fuzzy element (HFE).

Example 1. Let $X=\left\{x_{1}, x_{2}, x_{3}\right\}$ is the reference set, respectively, suppose that $h_{E}\left(x_{1}\right)=\{0.2,0.8\}$ $h_{E}\left(x_{2}\right)=\{0.4,0.5,0.7\}$ and $h_{E}\left(x_{3}\right)=\{0.4,0.6,0.8\}$ are the HFEs of $x_{i}(i=1,2,3)$ for a set $E$. Then $E$ can be considered as a HFS, i.e.

$$
E=\left\{\left\langle x_{1},\{0.2,0.8\}\right\rangle,\left\langle x_{2,}\{0.4,0.5,0.7\}\right\rangle,\left\langle x_{3,}\{0.4,0.6,0.8\}\right\rangle\right\} . \quad G_{i q 0}=\left\{\left(u_{i k}, v_{q d}\right) \mid u_{i k}-v_{q d}=0,\left(u_{i k}, v_{q d}\right) \in h_{i} \times h_{q}\right\}
$$

\section{B. Fuzzy Preference Relation}

Definition 2. [38] Let $R=\left(r_{i j}\right)_{n \times n}$ be a judgment matrix, then $R=\left(r_{i j}\right)_{n \times n}$ is fuzzy complementary judgment matrix if for any $i, j \in I$ satisfy

$$
r_{i j} \geq 0, r_{i j}+r_{j i}=1, r_{i i}=0.5
$$

Definition 3. [39] Let $R=\left(r_{i j}\right)_{n \times n}$ be a fuzzy complementary judgment matrix, then $R=\left(r_{i j}\right)_{n \times n}$ is fuzzy complementary judgment matrix with additive consistency if for any $i, j, k \in I$ satisfy

$$
r_{i j}=r_{i k}+r_{k j}-0.5
$$

Mean residual is defined as follow:

Definition 4. [40] For any complementary judgment matrix $R=\left(r_{i j}\right)_{n \times n}$, then $R=\left(r_{i j}\right)_{n \times n}$ is fuzzy complementary judgment matrix with additive consistency if exist standardization $\beta=\left(\beta_{1}, \beta_{2}, \ldots, \beta_{n}\right)^{T}$ and $c\left(c \geq \frac{n-1}{2}\right)$ for any $i, j \in I$ satisfy

$$
r_{i j}=c\left(\beta_{i}-\beta_{j}\right)+0.5
$$

By the formula (4) above, we can obtain by calculation

$$
\beta_{j}=\frac{1}{n}-\frac{1}{n c}\left(\sum_{i=1}^{n} r_{i j}-0.5 n\right) .
$$

\section{Hesitant Fuzzy PREFERENCE RELAtion AND It'S GENERALIZATION}

In this section, we will give definitions of mean residual and priority degree and prove it has additive consistency and then generalize its general model.

Definition 5. [41] Supposed two HFES $h_{i}=\left\{u_{i k}\right\}, i=1,2, \ldots, n, k=1,2, \ldots, l_{i}$;

$h_{q}=\left\{v_{q d}\right\}, q=1,2, \ldots, n, d=1,2, \ldots, l_{q}$. Let

$$
G_{i q}=\left\{\left(u_{i k}, v_{q d}\right) \mid u_{i k}-v_{q d}>0,\left(u_{i k}, v_{q d}\right) \in h_{i} \times h_{q}\right\}
$$

$$
\bar{G}_{i q}=\left\{\left(u_{i k}, v_{q d}\right) \mid u_{i k}-v_{q d}<0,\left(u_{i k}, v_{q d}\right) \in h_{i} \times h_{q}\right\}
$$

$$
D_{i q}= \begin{cases}\frac{1}{l_{i} l_{q}} \sum_{\left(u_{i k}, v_{q d}\right) \in G_{i q}}\left(u_{i k}-v_{q d}\right), & G_{i q} \neq \phi \\ 0, & G_{i q}=\phi\end{cases}
$$

$$
\bar{D}_{i q}= \begin{cases}\frac{1}{l_{i} l_{q}} \sum_{\left(u_{i k}, v_{q d}\right) \in \bar{G}_{i q}}\left(v_{q d}-u_{i k}\right), & \bar{G}_{i q} \neq \phi \\ 0, & \bar{G}_{i q}=\phi\end{cases}
$$

Where $D_{i q}$ denotes the average residual amount for $h_{i} \mathrm{f} h_{q} ; \bar{D}_{i q}$ denotes the average residual amount for $h_{q} \mathrm{f} h_{i}$. 
Definition 6. Let $D_{i q}$ denotes the average residual amount for $h_{i} \mathrm{f} h_{q} ; \bar{D}_{i q}$ denotes the average residual amount for $h_{q} \mathrm{f} h_{i}$. The priority degree $r_{i q}$ for $h_{i} \mathrm{f} h_{q}$ is defined by

$$
r_{i q}=\frac{1}{2 \max _{\forall i, q \in 1,2, \ldots, n}\left|D_{i q}-D_{q i}\right|}\left(D_{i q}-D_{q i}\right)+0.5
$$

Obviously, $D_{i q}=\bar{D}_{i q}, \quad r_{i q}$ denotes the degree of $h_{i} \mathrm{f} h_{q}$ in some extent. If $r_{i q}=1$, denotes $h_{i}$ over $h_{q}$ completely; If $r_{i q}=0.5$ denotes the priority degree for $h_{i}$ over $h_{q}$ is $0.5 ; \quad r_{i q}=0$ denotes $h_{q}$ over $h_{i}$ completely.

Property 1. $R=\left(r_{i q}\right)_{n \times n}$ is fuzzy complementary judgment matrix which has additive consistency.

Proof.

$$
\left|D_{i q}-D_{q i}\right| \leq \max _{\forall i, q \in 1,2, \ldots, n}\left|D_{i q}-D_{q i}\right|
$$

then

$$
\left|\frac{1}{2 \max _{\forall i, q \in 1,2, \ldots, n}\left|D_{i q}-D_{q i}\right|}\left(D_{i q}-D_{q i}\right)\right| \leq 0.5
$$

so $0 \leq r_{i q} \leq 1$.

Obviously,

$$
r_{i i}=0.5, r_{i q}+r_{q i}=1
$$

so, $R=\left(r_{i q}\right)_{n \times n}$ is fuzzy complementary judgment matrix.

Because,

$$
\begin{gathered}
D_{i m}-D_{m i}=D_{i m}-\bar{D}_{i m} \\
=\frac{1}{l_{i} l_{m}}\left[\sum_{\left(u_{i k}, g_{m r}\right) \in G_{i m}}\left(u_{i k}-g_{m r}\right)-\sum_{\left(u_{i k}, g_{m r}\right) \in \bar{G}_{i m}}\left(g_{m r}-u_{i k}\right)\right. \\
\left.+\sum_{\left(u_{i k}, g_{m r}\right) \in G_{i m 0}}\left(u_{i k}-g_{m r}\right)\right] \\
=\frac{1}{l_{i} l_{m}}\left[l_{m} \sum_{\left(u_{i k}, g_{m r}\right) \in G_{i m}} u_{i k}-l_{i} \sum_{\left(u_{i k}, g_{m r}\right) \in G_{i m}} g_{m r}\right.
\end{gathered}
$$

$$
\begin{gathered}
-l_{i} \sum_{\left(u_{i k}, g_{m r}\right) \in \bar{G}_{i m}} g_{m r}+l_{m} \sum_{\left(u_{i k}, g_{m r}\right) \in \bar{G}_{i m}} u_{i k} \\
\left.+l_{m} \sum_{\left(u_{i k}, g_{m r}\right) \in G_{i m 0}} u_{i k}-l_{i} \sum_{\left(u_{i k}, g_{m r}\right) \in G_{i m 0}} g_{m r}\right] \\
=\frac{1}{l_{i}} \sum_{k=1}^{l_{i}} u_{i k}-\frac{1}{l_{m}} \sum_{r=1}^{l_{m}} g_{m r}
\end{gathered}
$$

Similarly,

$$
D_{m q}-D_{q m}=\frac{1}{l_{m}} \sum_{r=1}^{l_{m}} g_{m r}-\frac{1}{l_{q}} \sum_{e=1}^{l_{q}} v_{q e}
$$

Then,

$$
\begin{aligned}
& r_{i m}+r_{m q}-0.5 \\
& =\frac{1}{2 \max _{\forall i, m \in 1,2, \ldots, n}\left|D_{i m}-D_{m i}\right|}\left(D_{i m}-D_{m i}\right) \\
& +\frac{1}{2 \max _{\forall m, q \in 1,2, \ldots, n}\left|D_{m q}-D_{q m}\right|}\left(D_{m q}-D_{q m}\right)+0.5 \\
& =\frac{1}{2 \max _{\forall i, q \in 1,2, \ldots, n}\left|D_{i q}-D_{q i}\right|}\left(D_{i m}-D_{m i}+D_{m q}-D_{q m}\right)+0.5 \\
& =\frac{1}{2 \max _{\forall i, q \in 1,2, \ldots, n}\left|D_{i q}-D_{q i}\right|}\left(\frac{1}{l_{i}} \sum_{k=1}^{l_{i}} u_{i k}-\frac{1}{l_{m}} \sum_{r=1}^{l_{m}}\right. \\
& \left.+g_{m r} \frac{1}{l_{m}} \sum_{r=1}^{l_{m}} g_{m r}-\frac{1}{l_{q}} \sum_{e=1}^{l_{q}} v_{q e}\right)+0.5 \\
& =\frac{1}{2 \max _{\forall i, q \in 1,2, \ldots, n}\left|D_{i q}-D_{q i}\right|}\left(D_{i q}-D_{q i}\right)+0.5 \\
& =r_{i q}
\end{aligned}
$$

So, the property 1 is proved.

Considering the complexity and uncertainty in the real world, we proposed the general model of hesitant fuzzy average residual. 
Definition 7. Supposed two $\mathrm{HFE}_{S}$ $h_{i}=\left\{u_{i k}\right\}, i=1,2, \ldots, n, d=1,2, \mathrm{~L}, l_{i}$;

$h_{q}=\left\{v_{q d}\right\}, q=1,2, \ldots, n, d=1,2, \ldots, l_{q}$. Let

$G_{i q}=\left\{\left(u_{i k}, v_{q d}\right) \mid u_{i k}-v_{q d}>0,\left(u_{i k}, v_{q d}\right) \in h_{i} \times h_{q}\right\}$

$\bar{G}_{i q}=\left\{\left(u_{i k}, v_{q d}\right) \mid u_{i k}-v_{q d}<0,\left(u_{i k}, v_{q d}\right) \in h_{i} \times h_{q}\right\}$

$G_{i q 0}=\left\{\left(u_{i k}, v_{q d}\right) \mid u_{i k}-v_{q d}=0,\left(u_{i k}, v_{q d}\right) \in h_{i} \times h_{q}\right\}$

General model average residual is defined as follow:

$$
\begin{array}{ll}
D_{i q}(\alpha)= \begin{cases}\frac{1}{l_{i} l_{q}} \sum_{\left(u_{i k}, v_{q d}\right) \in G_{i q}}\left(u_{i k}^{\alpha}-v_{q d}^{\alpha}\right), & G_{i q} \neq \phi \\
0, & G_{i q}=\phi\end{cases} \\
\bar{D}_{i q}(\alpha)= \begin{cases}\frac{1}{l_{i} l_{q}} \sum_{\left(u_{i k}, v_{q d}\right) \in \bar{G}_{i q}}\left(v_{q d}^{\alpha}-u_{i k}^{\alpha}\right), & \bar{G}_{i q} \neq \phi \\
0, & \bar{G}_{i q}=\phi\end{cases}
\end{array}
$$

where $\alpha>0, D_{i q}(\alpha)$ denote the average residual of $h_{i} \mathrm{f} h_{q}$ in general model; $\bar{D}_{i q}(\alpha)$ denote the average residual of $h_{q} \mathrm{f} h_{i}$ in general model.

Definition 8.Let $D_{i q}(\alpha)$ denotes the average residual amount for $h_{i} \mathrm{f} h_{q}$ in general model; $\bar{D}_{i q}(\alpha)$ denotes the average residual amount for $h_{q} \mathrm{f} h_{i}$ in general model. The priority degree $r_{i q}(\alpha)$ for $h_{i} \mathrm{f} h_{q}$ is defined by

$$
r_{i q}(\alpha)=\frac{1}{2 \max _{\forall i, q \in 1,2, \ldots, n}\left|D_{i q}(\alpha)-D_{q i}(\alpha)\right|}\left(D_{i q}(\alpha)-D_{q i}(\alpha)\right)+0.5
$$

where $\alpha>0, D_{i q}=\bar{D}_{i q}, \quad r_{i q}(\alpha)$ denote the priority degree of $h_{i} \mathrm{f} h_{q}$ of some extent in general model. If $r_{i q}(\alpha)=1$, denotes $h_{i}$ over $h_{q}$ completely; If $r_{i q}(\alpha)=0.5$, denotes the priority degree for $h_{i}$ over $h_{q}$ is $0.5 ; r_{i q}(\alpha)=0$ denotes $h_{q}$ over $h_{i}$ completely.

Example 2. Suppose that $X=\{x\} . H_{1}(x)=\{0.9,0.5\}$, $H_{2}(x)=\{0.8,0.7,0.3\} \quad$ and $\quad H_{3}(x)=\{0.8,0.5\} \quad$ are three $H F E_{S}$ on $X$.

From Definition 7, when $\alpha=0.1$, we have

$$
\begin{aligned}
& D_{12}(0.1)=0.0309, D_{21}(0.1)=0.0251 . \\
& D_{13}(0.1)=0.0170, D_{31}(0.1)=0.0112 . \\
& D_{23}(0.1)=0.0128, D_{32}(0.1)=0.0128 .
\end{aligned}
$$

From Definition 8, we have $r_{13}(0.1)=0.6602$, $r_{23}(0.1)=0.1602$.

Similarly, when $\alpha=1$, we have $r_{13}(1)=0.75, r_{23}(1)=0.25$.

when $\quad \alpha=10$, we have $r_{13}(10)=0.9657$, $r_{23}(10)=0.4657$.

when $\alpha=20$, we have $r_{13}(20)=0.9859$, $r_{23}(20)=0.4859$.

From this example, we learn about $\alpha$ take different value, $r_{i q}$ will change accordingly. When $\alpha$ is given a larger value, the value of $r_{i q}$ will more and more closer $0,0.5$ or 1 . If the value of $r_{i q}$ is close to 0.5 , we cannot know about the priority degrees between $H_{1}(x)$ and $H_{2}(x)$ clearly. Conversely, if the value of $r_{i q}$ is close to 0 or 1 , we can know about the priority degrees between $H_{1}(x)$ and $H_{2}(x)$ clearly. So the value of $\alpha$ will affect the order relations in some extent.

Property 2. $R(\alpha)=\left(r_{i q}(\alpha)\right)_{n \times n}$ is fuzzy complementary judgment matrix which has additive consistency.

Proof. The proof is similar to Theorem 1 and thus omitted.

\section{A New Approach to Hesitant Fuzzy Multiple ATTRIBUTE DECISION MAKING}

For a hesitant fuzzy multiple attribute decision making problem, let $A=\left\{A_{1}, A_{2}, \ldots, A_{s}\right\}$ be a set of alternatives and $C=\left\{C_{1}, C_{2}, \ldots, C_{t}\right\}$ be a set of attributes. Where $\omega=\left[\omega_{1}, \omega_{2}, \ldots, \omega_{t}\right]^{T}$ denotes the attribute weight vector satisfying $\sum_{j=1}^{t} \omega_{j}=1$ and $\omega_{j} \in[0,1], j=1,2, \ldots, t$. A group of expert provide possible membership degree of $A_{i}(i=1,2 \ldots, s)$ on $C_{j}(j=1,2 \ldots, t)$ and express as hesitant fuzzy element $h_{i j}$. The hesitant fuzzy decision matrix $H=\left(h_{i j}\right)_{s \times t}$ of $A$ on $C$ is 


$$
H\left[\begin{array}{cccc}
h_{11} & h_{12} & \ldots & h_{1 t} \\
h_{21} & h_{22} & \ldots & h_{2 t} \\
\mathrm{M} & \mathrm{M} & \mathrm{M} & \mathrm{M} \\
h_{s 1} & h_{s 2} & \ldots & h_{s t}
\end{array}\right]
$$

Definition 9. [42] For all of the $R_{c_{j}}(\alpha)$, a new hesitant fuzzy average operator is defined by

$$
W A_{M}\left(R_{C_{1}}(\alpha), R_{C_{2}}(\alpha), \ldots, R_{C_{t}}(\alpha)\right)=\sum_{j=1}^{t} \lambda_{j} R_{C_{j}}(\alpha)
$$

Where, $R_{C_{j}}(\alpha)=\left(r_{i q}^{C_{j}}(\alpha)\right)_{s \times s}, \quad \alpha>0, \lambda_{j}>0$, $r_{i q}^{C_{j}}(\alpha)$ denote the priority degree of $h_{i} \mathrm{f} h_{q}$ for attribute $C_{j}$ in general model.

Definition 10. [42]

Let

$$
\bar{R}(\alpha)=\left(\bar{r}_{i q}(\alpha)\right)_{s \times s}=\sum_{j=1}^{t} \omega_{j} R_{C_{j}}(\alpha)
$$

$\bar{R}(\alpha)$ denote the fuzzy complementary judgment matrix of $h_{i} \mathrm{f} h_{q}$ on the basis of attribute weighting $\omega_{j}$ in general model. Where $\alpha>0, \sum_{j=1}^{t} \omega_{j}=1$ and $\omega_{j} \in[0,1]$, $R_{C_{j}}(\alpha)=\left(r_{i q}^{C_{j}}(\alpha)\right)_{s \times s}, r_{i q}^{C_{j}}(\alpha)$ denote the priority degree of $h_{i} \mathrm{f} h_{q}$ for attribute $C_{j}$ in general model.

Property 3. [42] Suppose $R(\alpha)=\left(r_{i q}(\alpha)\right)_{n \times n}$ is fuzzy complementary judgment matrix which has additive consistency, then $\bar{R}(\alpha)=\left(\bar{r}_{i q}(\alpha)\right)_{s \times s}=\sum_{j=1}^{t} \omega_{j} R_{C_{j}}(\alpha)$ satisfied $\sum_{j=1}^{t} \omega_{j}=1$ and $\omega_{j} \in[0,1], j=1,2 \ldots, t$ is also fuzzy complementary judgment matrix which has additive consistency. have

Proof. From theorem 2, Definition 8 and Definition 9, we

$$
\bar{r}_{i q}(\alpha)=\sum_{j=1}^{t} \omega_{j} r_{i q}^{C_{j}}(\alpha)=\sum_{j=1}^{t} \omega_{j}\left(r_{i p}^{C_{j}}(\alpha)+r_{p q}^{C_{j}}(\alpha)-0.5\right)
$$

$$
\begin{gathered}
\left.=\sum_{j=1}^{t} \omega_{j} r_{i p}^{C_{j}}(\alpha)+\sum_{j=1}^{t} \omega_{j} r_{p q}^{C_{j}}(\alpha)-0.5\right) \\
=\bar{r}_{i p}(\alpha)+\bar{r}_{p q}(\alpha)-0.5 \quad i, p, q=1,2, \ldots, s
\end{gathered}
$$

$$
\text { Obviously, } \quad r_{i i}=0.5 \quad, \quad 0 \leq \bar{r}_{i q}(\alpha) \leq 1 \quad \text {, }
$$
$\bar{r}_{i q}(\alpha)+\bar{r}_{q i}(\alpha)=1$.

So, the theorem is proved.

In the following, a new method based on the fuzzy complementary judgment matrix is proposed to solving the multiple attribute decision making problems, in which the information about attribute weights is given, and the attribute values take the form of hesitant fuzzy element, the new approach involves the following steps:

Step 1: Let $H=\left(h_{i j}\right)_{s \times t}$ be a hesitant fuzzy decision-making matrix, where $h_{i j}$ of each alternative $H_{i} \in H \quad$ of each attribute $\quad C_{j} \in C \quad$ and $\omega=\left(\omega_{1}, \omega_{2}, \ldots, \omega_{t}\right)^{T}$ be the weight vector of attributes, where $\omega_{j} \in[0,1], j=1,2, \ldots, t$, and $\sum_{j=1}^{t} \omega_{j}=1$.

Step 2: $\mathrm{Eq}(36)$ is used to calculate the fuzzy complementary judgment matrix $R_{C_{j}}(\alpha), C_{j} \in C$.

Step 3: Utilize the $\mathrm{Eq}(42)$ calculate the weighted fuzzy complementary judgment matrix $\bar{R}(\alpha)$.

Step 4: $\mathrm{Eq}(5)$ is used to calculate the weight $\beta_{i},(i=1,2,3,4)$ of each alternatives.

Step 5: Rank all the alternatives $A_{i},(i=1,2, \ldots, s)$ Select the best alternative(s).

Step 6: End.

\section{ILLUSTRATIVE EXAMPLE}

In this section, a numerical example is given to show the feasibility and validity of the proposed the $W A_{M}$ operator. We analyze an investment selection multiple attribute decision making problem.

Example [43]. A board of directors of an enterprise consists of five numbers, and they are planning to invest a sum of money in a suitable project of huge potential over the next 5 years. Suppose four possible projects $A=\left(A_{1}, A_{2}, A_{3}, A_{4}\right)$ will be evaluated by the board of directors. To evaluateand rank these projects, four criteria are suggested by the Balanced Score card methodology (it should be noted that all of them are of the maximization type), that is, $C_{1}$ : financial perspective, $C_{2}$ : 
the customer satisfaction, $C_{3}$ : internal business process perspective, and $C_{4}$ : learning and growth perspective. The weight vector of the criteria is given as
$\omega=(0.2,0.3,0.15,0.35)^{T}$. Assessment values given by the decision makers are displayed in the hesitant fuzzy decision matrix in Table 1.

TABLE I. HESITANT FUZZY DECISION MATRIX

\begin{tabular}{lllll}
\hline & $C_{1}$ & $C_{2}$ & $C_{3}$ & $C_{4}$ \\
\hline$A_{1}$ & $\{0.2,0.4,0.7\}$ & $\{0.2,0.6,0.8\}$ & $\{0.2,0.3,0.6,0.7,0.9\}$ & $\{0.3,0.4,0.5,0.7,0.8\}$ \\
$A_{2}$ & $\{0.2,0.4,0.7,0.9\}$ & $\{0.1,0.2,0.4,0.5\}$ & $\{0.3,0.4,0.6,0.9\}$ & $\{0.5,0.6,0.8,0.9\}$ \\
$A_{3}$ & $\{0.3,0.5,0.6,0.7\}$ & $\{0.2,0.4,0.5,0.6\}$ & $\{0.3,0.5,0.7,0.8\}$ & $\{0.2,0.5,0.6,0.7\}$ \\
$A_{4}$ & $\{0.3,0.5,0.6\}$ & $\{0.2,0.4\}$ & $\{0.5,0.6,0.7\}$ & $\{0.8,0.9\}$ \\
\hline
\end{tabular}

Step 1: Construct a hesitant fuzzy decision-matrix $H=\left(h_{i j}\right)_{s \times t}$ according to the decision information given by the experts as Table 1.

Step 2: $\mathrm{Eq}(36)$ is used to calculate the fuzzy complementary judgment matrix $R_{C_{j}}(\alpha), C_{j} \in C$ as follows:

$$
\begin{aligned}
& R_{C_{1}}(1)=\left[\begin{array}{cccc}
0.5 & 0 & 0.1075 & 0.3573 \\
1 & 0.5 & 0.6071 & 0.8569 \\
0.8925 & 0.3929 & 0.5 & 0.7498 \\
0.6427 & 0.1431 & 0.2502 & 0.5
\end{array}\right] \\
& R_{C_{2}}(1)=\left[\begin{array}{cccc}
0.5 & 0.9998 & 0.7320 & 1 \\
0.0002 & 0.5 & 0.2322 & 0.5 \\
0.2680 & 0.7678 & 0.5 & 0.7678 \\
0 & 0.5 & 0.2322 & 0.5
\end{array}\right] \\
& R_{C_{3}}(1)=\left[\begin{array}{cccc}
0.5 & 0.4167 & 0.2083 & 0 \\
0.5833 & 0.5 & 0.2917 & 0.0833 \\
0.7917 & 0.7083 & 0.5 & 0.2917 \\
1 & 0.9167 & 0.7083 & 0.5
\end{array}\right] \\
& R_{C_{4}}(1)=\left[\begin{array}{cccc}
0.5 & 0.2714 & 0.5571 & 0.0571 \\
0.7286 & 0.5 & 0.7857 & 0.2857 \\
0.4429 & 0.2143 & 0.5 & 0 \\
0.9429 & 0.7143 & 1 & 0.5
\end{array}\right]
\end{aligned}
$$

Step 3: Utilize the Eq(42) calculate the weighted fuzzy complementary judgment matrix $\bar{R}(\alpha)$ as follows:

$$
\bar{R}(1)=\left[\begin{array}{cccc}
0.5 & 0.4574 & 0.4673 & 0.3914 \\
0.5426 & 0.5 & 0.5098 & 0.4339 \\
0.5327 & 0.4902 & 0.5 & 0.4241 \\
0.6086 & 0.5661 & 0.5759 & 0.5
\end{array}\right]
$$

Step 4: $E q(5)$ is used to calculate the weight $\beta_{i},(i=1,2,3,4)$ of each alternatives as follows:

$$
\begin{aligned}
\beta_{1}=0.2270, \beta_{2} & =0.2483, \quad \beta_{3}=0.2434, \\
\beta_{4} & =0.2813 .
\end{aligned}
$$

Step 5: Rank all the alternatives $A_{i},(i=1,2, \ldots, s)$, Select the best alternative(s):

$$
A_{4} \mathrm{f} \quad A_{2} \mathrm{f} \quad A_{3} \mathrm{f} A_{1} \text {. }
$$

Step 6: End.

\section{CONCLUSIONS}

In this paper, we constructed fuzzy complementary judgment matrix which has additive consistency and then we generalized it to the general model. Based on the presented fuzzy complementary judgment matrix and hesitant fuzzy average operator, a new multiple attribute decision making approach was obtained. Finally, an illustrative example was given to show the rationality of the proposed method.

\section{ACKNOWLEDGMENTS}

The authors want to thank the anonymous referees for the valuable comments that have led to an improvement in this paper. This research was supported by the National Foundation of China (No.71761001,71761002) and Guangxi Colleges and Universities Key Laboratory of Mathematics and Its Applications.

\section{REFERENCES}

[1] Zadeh L. A. Fuzzy sets. Inform Contr 1965;8:338-356. 
[2] Zadeh L. A. The concept of a linguistic variable and its application to approximate reasoning-1. Inf Sci 1975;8:199-249.

[3] Dubois D, Prade H. Fuzzy sets and systems: theory and applications. New York: Academic Press 1980.

[4] Yager R. R. On the theory of bags. Int J Gen Syst 1986;13:23-37.

[5] Atanassov K. Intuitionistic fuzzy sets. Fuzzy Sets Syst 1986;20:87-96.

[6] Xu Z. S, Xia M. M. Distance and similarity measures for hesitant fuzzy sets. Inf Sci 2011a;181:2128-2138.

[7] Torra V. Hesitant fuzzy sets. Int J Intell Syst 2010;25: 529-539.

[8] Qin J. D, Liu X. W, Pedrycz W. Frank aggregation operators and their application to hesitant fuzzy muultiple attribute decision making. Appl Soft Comput 2016;41:428-452.

[9] Jin F, Ni Z. W, Chen H. Y. Note on "hesitant fuzzy prioritized operators and their application to multiple atribute decision making". Knowl-Based Syst 2016;96:115-119.

[10] Zhu B, Xu Z. S, Xia M. M. Hesitant fuzzy geometric Bonfreroni means. Inf Sci 2012a;205:72-85.

[11] Li D, Zeng W, Li J. New distance and similarity measures on hesitant fuzzy sets and their applications in multiple criteria decision making. Eng Appl Artif Intell 2015a;40:11-16.

[12] Li D, Zeng W, Zhao Y. Note on distance measure of hesitant fuzzy sets. Inf Sci 2015b;321:103-115.

[13] Dgenci M. A new distant measure for interval valued intuitionistic fuzzy sets and its application to group decision making problems with incomplete weihgts information. Appl Soft Comput 2016;41:120-134.

[14] Xia M. M, Xu Z. S. Hesitant fuzzy information aggregation in decision making. Int J Approx Reason 2011;52:395-407.

[15] Xu Z. S, Xia M. M. Hesitant fuzzy entropy and cross-entropy and their use in multiattribute decision making. Int J Intell Syst 2012;27:799-822.

[16] Xu Z. S, Xia M. M. On distance and correlation measures of hesitant fuzzy information. Int J Intell Syst 2011;26:410-425

[17] Wei G. Hesitant fuzzy prioritized operators and their applicaton to multiple attribute decision making. Knowl-Based Syst 2012;31:176-182.

[18] Xia M. M, Xu Z. S, Chen N. Some hesitant fuzzy aggregation operators with their application in group decision making. Group Decis Negot 2013;22:259-279.

[19] Zhang Z. M. Hesitant fuzzy power aggregation operators and their application to multiple attribute group decision making. Inf Sci 2013;234:150-181.

[20] Farhadinia B. Information measures for hesitant fuzzy sets and interval valued hesitant fuzzy sets. Inf Sci 2013;240:129-144.

[21] Farhadinia B. A novel method of ranking hesitant fuzzy values for multiple attribute decision-making problems. Int $\mathrm{J}$ Intell Syst 2013;28:752-767.

[22] Zhang Z. M, Wu C. On the use of multiplicative consistency in hesitant fuzzy linguistic preference relations. Knowl-Based Syst 2014;72:13-27.

[23] Zhu B. Consistency measures for hesitant fuzzy linguistic preference relations. Fuzzy Systems 2014;22:35-45.

[24] Zhang Y. X, Xu Z. S, Wang H, Liao H. C. Consistency-based risk assessment with probabilistic linguistic preference relation. Applied Soft Computing 2016;49:817-833.

[25] Tan J. Y, Zhu C. X, Zhang X. Z, Zhu L. The method of hesitant fuzzy multiple attribute decision making based on group consistency. Opeerations Research and Management Science 2016;25:105-109.

[26] Qin J. D, Liu X. W, Pedrycz W. Frank aggregation operators and their application to hesitant fuzzy multiple attribute decision making. Applied Soft Computing 2016;41:428-452.

[27] Zhu C. X, Zhu L, Zhang X. Z. Linguistic hesitant fuzzy power aggregation operators and their applications in multiple attribute decision-making. Inf Sci 2016;367-368:809-826.

[28] Jin F. F, Ni Z. W, Chen H. Y. Note on "Hesitant fuzzy prioritized operators and their application to multiple attribute decision making". Knowl-Based Syst 2016;96:115-119.

[29] Lan J. B, Jin R. F, Zheng Z. Y, Hu M. M. Priority degrees for hesitant fuzzy sets: Application to multiple attribute decision making. Operations Research Perspectives 2017;4:67-73.
[30] Tang X. A, Fu C, Xu D. L, Yang S. L. Analysis of fuzzy Hamacher aggregation functions for uncertain multiple attribute decision making. Inf Sci 2017;387:19-33.

[31] Joshi D, Kumar S. Interval-valued intuitionistic hesitant fuzzy Choquet integral based TOPSIS method for multi-criteria group decision making. European Journal of Operational Research 2015;248:183-191.

[32] Zhang Y. J, Xie A, Wu Y. T. A hesitant fuzzy multiple attribute decision making method based on linear programming and TOPSIS IFACPapersOnline 2015;48:427-431.

[33] Xu Z. S, Zhang X. L. Hesitant fuzzy multi-attribute decision makingbased on TOPSIS with incomplete weight information. Knowl-Based Syst 2013;52:53-64.

[34] Qin J. D, Liu X. W, Pedrycz W. An extended VIKOR method based on prospect theory for multiple attribute decision making under interval type-2 fuzzy environment. Knowl-Based Syst 2015;86:116-130.

[35] Wu Z. B, Ahmad J, Xu J. P. A group decision making framework based on fuzzy VIKOR approach for machine tool selection with linguistic information. Applied Soft Computing 2016;42:314-324.

[36] Sun G. D, Guan X, Yi X, Zhou Z. Grey relational analysis between hesitant fuzzy sets with applications to pattern recognition. Expert Systems with Applications 2018;92:521-532.

[37] Zhang $\mathrm{X}$, Jin F, Liu P. D. A grey relational projection method for multiattribute decision making based on intuitionistic trapezoidal fuzzy number. Applied Mathematical Modelling 2013;37:3467-3477.

[38] Orlovski S. A. Decision-making with a fuzzy preference relation. FuzzySets Syst 1978;1:155-167.

[39] Tanino T. Fuzzy preference orderings in group decision making. FuzzySets Syst 1984;12:117-131.

[40] Lv Y. J.Weight calculation method of fuzzy analytical hierarchy process.Fuzzy Systems and Mathematics 2002;16:79-85.

[41] Lan J. B, Jin R. F, Zheng Z.Y, Hu M. M. Priority degrees for hesitant fuzzy sets: Application to multiple attribute decision making. Oper ResPersp 2017;4:67-73.

[42] Xu Z. S. Uncertain multiple attribute decision making: Methods and applications. Beijing: Peking University Press 2004:22-23.

[43] Zhang N, Wei G. W. Extension of vikor method for decision making problem based on hesitant fuzzy set. Appl Math Model 2013;37:4938-4947. 